\title{
Research for Emergency Exercise Network System based on Scenario Analysis
}

\author{
JiAn Sun ${ }^{1, \mathrm{a}}$, Guoqiang Cai ${ }^{1, \mathrm{~b}}$, Yatao Wang ${ }^{2}$, Kun Zhang $^{3}$,Hui Zhen ${ }^{4}$ \\ Beijing Jiaotong University, State Key Lab of Rail Traffic Control \\ And Safety, Beijing100044, China; \\ bguoqiangcai@163.com
}

\begin{abstract}
.
Nowadays, quite a few accidents occur around us. During an emergency situation in public, decisions must be taken in a timely manner. But seldom people have the experiences to deal with such hard situations. So, there is a great need of a simulate system based on network for emergency exercise. This Emergency Exercise System based on Scenario Analysis use Go-uncertain technology to deduce emergency situations and use Multi-agent technology to realize network communications between agents. Exercising in such simulate emergency situations, participators can constantly strengthen the capacity of disposing emergency events.
\end{abstract}

Keywords: Emergency Exercise, Scenario Analysis, Simulation, Network, Agent, Go-uncertain

\section{Background}

3DGIS Technology developed in various industries at home and abroad [1].C3D three dimensional GIS platform as the core, combine VS2003 and DirectX, forming a well blowout accident emergency exercise based on GIS visualization system [2]. [1,2] and this Emergency Exercise System based on Scenario Analysis in system concept, structure, user groups, function distribution, are different. In auxiliary of evacuation simulation software, research evacuates efficiency influenced by objective environment factors and micro individual factors [3]. Design out any logic personnel evacuation model and stated modeling thought and process; through analysis simulation statistics get of personnel time of evacuation [4].On net logo platform established a simulation 
system, on a four layer building of scene for built model and simulation [5].

Research harmful gas leaked and cause of injury [6]. [3,4,5,6].and this Emergency Exercise System is different, which based on Automatically Deduction Engine and Multi-task Interactive Exercises and Network Communications Fault-tolerant and Multi-agent Management technology, can achieved "Guide", "Education", "Learning", "Play" ,"Evaluation".

\section{Architecture}

This emergency interactive exercise system, based on Indeterminate Deduction Theory and Rule Task Theory and Auto-adapted System Theory, supported by Automatically Deduction Technology and Interactive Exercise Technology and Communications Fault-tolerant Technology and Parallel Simulation Technology and Multi-Agent Technology, could set basic scenes, establish Rules Scene Library, assess Model Knowledge database; on scene platform this system start from basic scene to do interactive exercise and provide interactive platforms for performers. Director group of performers into different roles according to the actual situation and coordinate divisions in the training process. This system enhances the participator's awareness of crisis management and enriches their experiences of emergency disposal.

Innovation of this system is using the SECA exercise practice rules, uncertainty analysis; emergency scenarios task analysis method to construct the scenario emergency exercise platform which realizes "Guide", "Education", "Learning", "Play","Evaluation" functions. This system has evaluated mechanisms which can find performers' lack in dealing with emergencies and propose skills and exercise approaches for improvement. In addition, this project has the advantages of many scenes, multi interactive, so in emergency exercises have played a great role in promoting.

"Guide" - according to different characteristics of the participator's focus, work by built in material database and process designer, customizing the new curriculum, as well as modify existing courses.

"Education" - the teacher can use instructors' control class of processes in the 
system and at any time according to the students ' reactions into the event leads the discussion to achieve teaching objectives.

"Learning" - a participant could use the system to experience disaster sites and pressure from the emergency contingency and other relevant affairs.

"Play" - student groups to simulate different roles in different sectors, through an interactive discussion using simulation exercise system truly deduction, to experience the true meaning of the disposal process and see the result.

"Evaluation" - instructors and experts through the disposal process of the students replay to gives a reasonable assessment, in order to achieve optimal results, the participants ' disposition is preserved, as accumulation.Fig. 1 has showed the Architecture of this system.

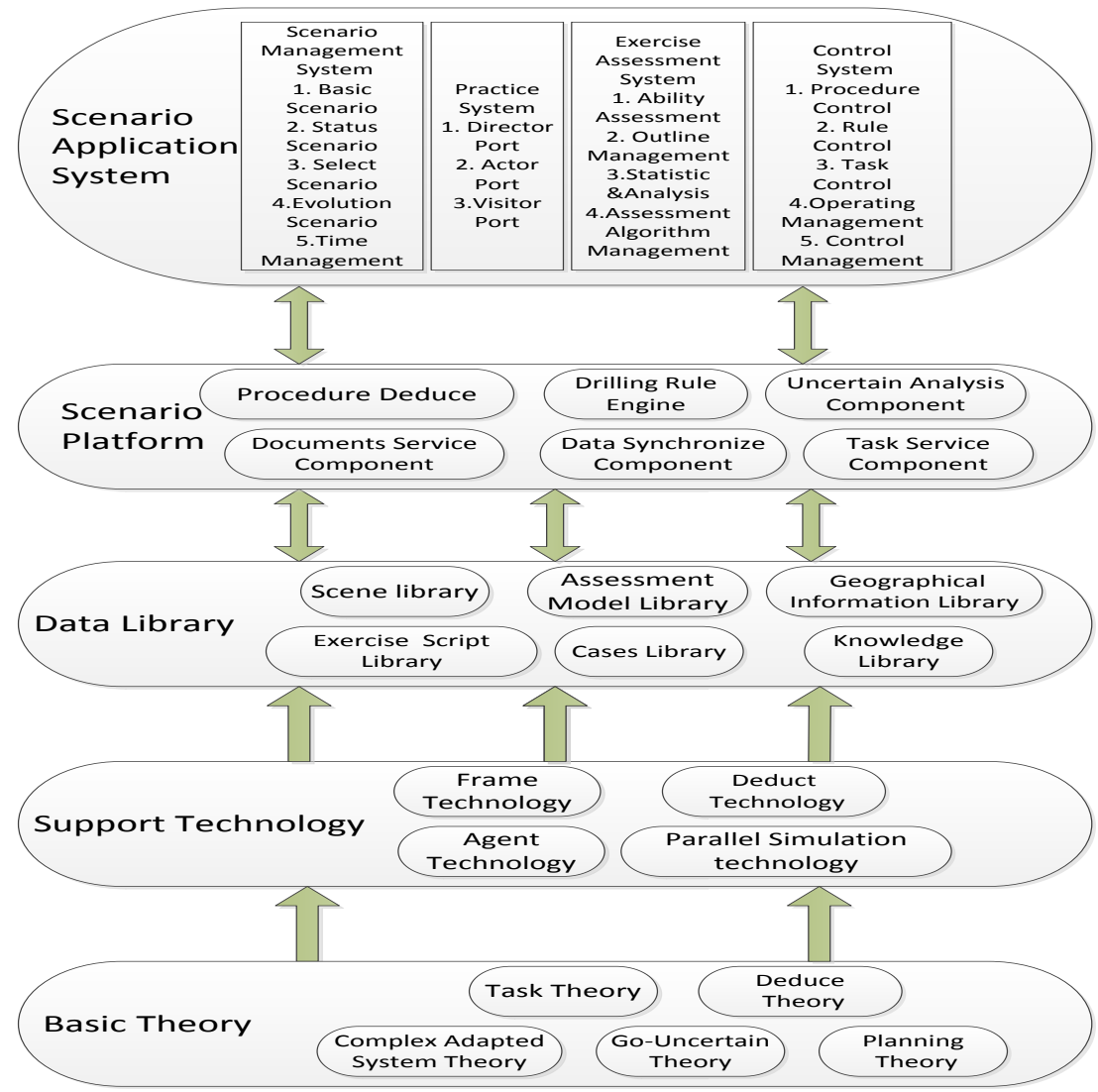

Fig.1 Emergency Exercise System Architecture 
This system dominated by scene simulation exercises, combining lectures, case teaching and event rehearsal-style diversified teaching patterns, professional emergency management training and software application systems, with the accumulation of knowledge, support for multiple role scenario simulation complex training content and easy customization features.

This project designed primarily to implement the following functions:

1) Online edit courses: courses are edited by various directors who logged on remote editing system and courseware can be shared with other directors.

2) Online learning system: provides audio/video e-learning training courses, participants can log on the website and learn emergency management knowledge and experience unexpected incidents and disposal process.

3) Longitudinal assessment: records student's participation in the course of study, to evaluate the trainee's interaction solutions.

4) Transverse interaction: implementing information transfer between participating students coordinates the development of solutions.

5) Online exam: emergency response platform provides various emergency management quiz, students can log on to the system, the system automatically graded according to answers of students

6) Material library: upload, download, share all kinds of training and demonstration materials and provide the Director with a variety of different scenarios.

7) Training program: for recent training programs and classes, and contact information.

8) Role management: role is participant of the exercise and the executive Body, according to the different exercise topics; you should choose the appropriate roles. Role types are divided into the rescue class, command class and observation class. Different roles have different skills and equipment, when exercises start to execute, role through the use of specific skills and equipment to accomplish the task.

9) Student information management and personal information management. 
10) Faculty of information management, including teachers basic information, teaching and so on.

11) Experts in information management, including reviews of experts.

\section{Key Technology}

1. Automatic Deduction Technology based on GO-uncertain engine When director set the automatic pre-designed scripted properties, the scripts support automatic deduction. Without Director-end intervention, you can complete the preset script's deductive process. When the automatic deduction, Director-side are ready to intervene and take over the rehearsal process to meet exercise control requirements. In event occurred, many indeterminate factors appeared, event of development does not according to a fixed mode or direction. Due to indeterminate factors analysis belongs to timing problem, so need to analysis various state by time sequence according to event occurred, so reference GO method and accident tree model combination, formed integrated analysis GO-uncertain method which make quantitative analysis on indeterminate factors.

GO-Uncertain method uses time series as axis of scenario, use basic unit models and logical construct models and compute the probability parameters according to certain rules and deduce combined with uncertainty events in real time emergency situations.

2. Multi-agent management technology based on network

In an interactive exercise, performers and directors need complex analysis of the information for decision-making, while processing time is very precious, it is impossible to make each participator instant searching on Internet. This system using Multi-agent technology based on network, from "people looking for information" to "information looking for people". It sets each participator staff as an Agent and integrates user's resource and guide users to find, saving user's time and making the tasks of handling efficiency. This system uses the B/A/S model (Browser/Agent/server) which is a "thin 
client" mode. And the client browser (such as Netscape or Internet Explorer) could access internet conveniently, eliminating the need for different customers for different client installation program, this way provides a unified environment for complex distributed applications. Middle Agents layer responsible for the network communication between client and server, and servers needs to run protocols such as MIME and HTTP to support network communication. In this structure, the database user does not interact directly with the database server, such way improves the security of the database side. System integrated framework based on network is shown in the Fig. 2

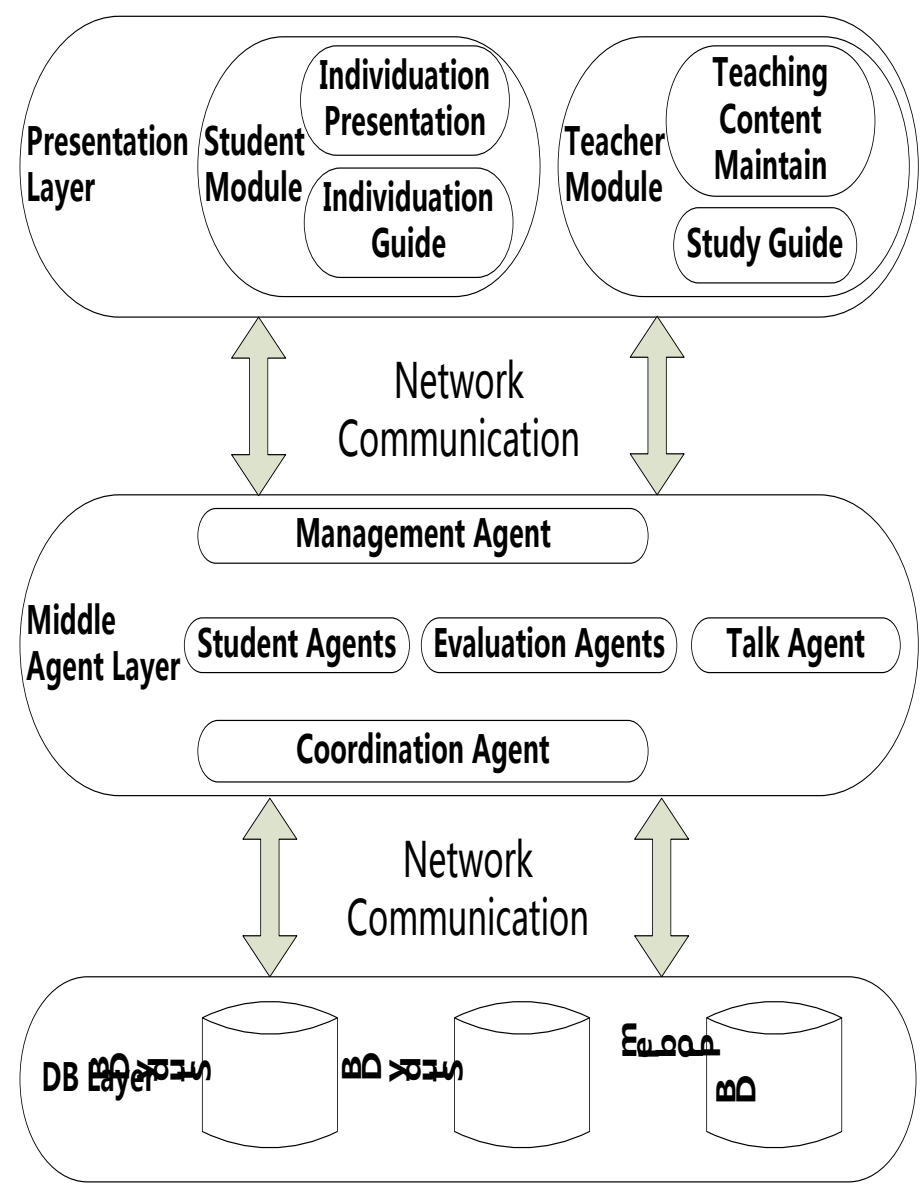

Fig.2 Architecture of Multi-agent based on network. 


\section{Conclusion}

This Emergency Interactive Exercise System based on Automated Deduction Engine, Multi-mission Interactive Exercise (SECA Exercise rules), to realize exercise mechanism based on GO-uncertain. This system can put to use in all level of organizations and administrations, to practice and enhance their ability in dealing with unexpected hard situations. When accident occurs, relevant persons could react calmly and reduce the loss of the accident to a minimum.

\section{Acknowledgements}

The author thanks the anonymous reviewers for their useful comments. This paper was supported by the Beijing Jiaotong University and the State Key

Laboratory of Rail Traffic Control and Safety project No.(I12B300051(J00ZZ120424)) and No.(I09K00030(RCS2008ZZ004)) and No.( I12B200020 ( 2011AA110501-1-1)).

\section{References}

[1] Renjun Fu, Design and Implementation of Electric Power Industry emergency drills system based on 3DGIS technology [d]. Beijing University of technology, 2012.

[2] Qian Liu, Research and realization of Blowout Visual Emergency Exercise System based on GIS [d]. Hubei University, 2011.

[3] Meng Yuan,Research of microcosmic characteristic analysis and simulation based on Multi-Agent, in Emergency evacuation for large-scale public buildings [d]. Harbin Engineering University, 2012.

[4] Libing Yang, Emergency evacuation systems and simulation in public places [d]. Central South University, 2009.

[5] Chen Qi,Study and Application of PSO algorithm in crowds evacuate simulation [d]. Sun Yat-sen University, 2011.

[6] Lin Shui, Emergency management system based on gas diffuse simulation 
[d]. Chongqing University, 2012.

[7] Yatao wang; Guoqiang Cai; Xinyu Wu, The Application of Multi-Agent in Emergency Events of Metro Station, Applied Mechanics and Materials Vols. 644-650 (2014) pp 2699-2702

[8] Roberto A; Flores-Mendez, Towards the Standardization of Multi-Agent Systems Architectures: An Overview, ACM Crossroads' special issue on Intelligent Agents, summer 1999. 